Research Article

\title{
Bispectral-Based Ultrasound Image Restoration Algorithm for Neurological Disorders Analysis in Patients Anesthetized with Sevoflurane
}

\author{
Yanfang Lou $(\mathbb{D}$, Bing Liang $(\mathbb{D}$, Qing Gu $(\mathbb{D}$, and Zhiwei He \\ Pediatric Hospital Affiliated to Fudan University, Shanghai 201102, China \\ Correspondence should be addressed to Zhiwei He; louyf_@fudan.edu.cn
}

Received 31 March 2021; Revised 6 May 2021; Accepted 2 June 2021; Published 12 June 2021

Academic Editor: Gustavo Ramirez

Copyright (c) 2021 Yanfang Lou et al. This is an open access article distributed under the Creative Commons Attribution License, which permits unrestricted use, distribution, and reproduction in any medium, provided the original work is properly cited.

\begin{abstract}
The aim of this work was to investigate preventive effect of dexmedetomidine combined with sevoflurane on neurological disorders in liver cancer patients during postoperative recovery under the ultrasound image restoration (UIR) algorithm. A blind deconvolution medical UIR algorithm was established in this study. The patients anesthetized with sevoflurane for liver cancer surgery were rolled into an experimental group (group A) (dexmedetomidine treatment before surgery) and a control group (group B) (normal saline treatment before surgery). They were monitored routinely before, during, and after surgery, and immune and liver functions of patients were observed before and after surgery. Besides, their cognitive function and adverse reactions were assessed after surgery. Results showed that patients from group A had smaller fluctuation in average arterial blood pressure and heart rate, but their bispectral index (BIS) increased obviously after surgery in contrast to those of group B $(P<0.05)$. Moreover, changes of immune and liver functions in patients from group A decreased sharply compared with group B $(P<0.05)$. The postoperative minimental state examination (MoCA) score of patients from group A was $27.51 \pm 2.02$, while the score of group B was $25.79 \pm 2.35$. The postoperative (MoCA) score of group A was markedly higher than the score of group B, and the difference was statistically obvious $(P<0.05)$. Besides, the incidence of postoperative cognitive dysfunction (POCD) in patients from group $A$ and $B$ was $12.91 \%$ and $32.26 \%$ in turn. The incidence of POCD in patients from group A was sharply lower than the incidence of group $\mathrm{B}(P<0.05)$. In addition, the total incidence of adverse reactions in patients from group A was $6.45 \%$, which was significantly lower than the $22.58 \%$ of group $B$, and the difference was statistically substantial $(P<0.05)$. In summary, dexmedetomidine combined with sevoflurane could effectively reduce POCD and promote systemic circulation recovery, which had clinical application value.
\end{abstract}

\section{Introduction}

Liver cancer is a malignant tumor of the liver, which can be divided into primary and secondary liver cancers. What is more, primary liver cancer is the most dangerous malignant tumor in the world, mainly including hepatocellular carcinoma. The total morbidity and fatality rate rank among the top of the world's diseases [1]. The early onset of liver cancer is relatively insidious, and there is lack of specific symptoms in clinical, so many patients miss the best treatment time. The first choice for liver cancer in China is still surgery [2].
Sevoflurane is a new inhalation anesthetic with excellent physical properties. Its blood gas fraction coefficient is only 0.59 , it can induce rapidly with less tissue uptake, and patients wake up faster. The metabolism of sevoflurane in the body is mainly hydrolyzed by the cytochrome P-450 system, which has little effect on circulation inhibition and myocardium conducting system. The low concentration of sevoflurane can make the heart, ejection, and contraction rates of patients drop rapidly. But it should not be applied in patients with elevated intracranial pressure. In addition, sevoflurane has a strong inhibitory effect on breathing [3]. Dexmedetomidine is 
a kind of $\alpha 2$ adrenergic receptor agonist that is highly selective, which is featured with fast onset, short action, sedation, analgesia, and inhibition of sympathetic nerve activity, stable hemodynamics, less anesthetic dosage, and no respiratory depression. When dexmedetomidine achieves and maintains a sufficient level of sedation, it is easy to wake patients up and can reduce the amount of other sedative drugs. The sedation induced by dexmedetomidine is similar to nonrapid eye movement sleep so that the quality of sleep can be improved, and dexmedetomidine is beneficial to the recovery of the immune system [4-6].

Anesthesia recovery period refers to the recovery period of patients with anesthesia after surgery, and surgical trauma, anesthesia drugs, and patient's own condition make the complications of anesthesia recovery period more common, which will affect the postoperative recovery status of patients $[7,8]$. Improper handling can endanger the life and health of patients. The vegetative system dysfunction means a series of symptom groups caused by the imbalance of the functions innervated by vegetative nerves. Its clinical manifestations can involve multiple systems throughout the body, often accompanied by emotional changes such as anxiety, tension, and depression [9]. Ultrasound imaging is to apply ultrasound beams to scan the body, so as to acquire the images of internal organs through the reception and processing of reflected signals. Ultrasound imaging is often employed to determine the location, size, and morphology of the lesion to provide tissue anatomy maps. It has a wide range of applications in obstetrics and gynecology, cardiovascular system, and digestive system. Besides, ultrasound imaging has the advantages of lossless-ness, portability, safety, low cost, and real-time imaging, which is one of the commonly applied methods in medical diagnosis. However, the resolution of ultrasound images is low, and the influence of noise is relatively large. The noise mainly comes from the accumulation of point spread function and tissue reflectance convolution [10]. With the research of digital image technology, the application of digital images to ultrasound images can promote the image quality. The image restoration algorithm is used for correction of atmospheric effects and geometry on remote sensing image data and amendment of scanning line leakage and misalignment caused by equipment. The above is the process of reconstructing a low-quality image into an ideal and high-quality image that is close to or completely degraded [11].

To sum up, the blind deconvolution UIR was applied in the image analysis of liver cancer patients to improve the quality of ultrasound images in order to enhance the efficiency of doctors' diagnosis of the disease. Moreover, the combination of dexmedetomidine and sevoflurane was adopted in the surgery of patients with liver cancer. Various physiological indexes of the patients were detected during the surgery, so as to judge the preventive effect of neurological disorders in patients at the recovery period.

\section{Experimental Methods}

2.1. Establishment of Research Data. 64 patients with caudate lobe liver cancer were selected as the research subjects, who were hospitalized from October 2019 to October 2020 in hospital and underwent surgical treatment. The criteria for inclusion were defined to include patients who were diagnosed as caudate lobe liver cancer through abdominal ultrasound, needed to receive the surgical treatment, were 40-70 years old, and weighed between 50 and $75 \mathrm{~kg}$. There were no obvious differences in the gender, age, height, weight, surgical time, American Society of Anesthesiologists (ASA) classification, and bleeding volume among patients from the two groups $(P>0.05)$. The criteria for exclusion were defined to include patients who had sinus bradycardia or malignant arrhythmia before surgery, suffered from severe respiratory dysfunction, and took corticosteroids or immunosuppressive agents recently.

All subjects and their families had signed the informed consent forms, and all patients were rolled into two groups (group A and B). The patients from both groups were anesthetized with sevoflurane during surgery. The patients from group A were given with a dose of dexmedetomidine hydrochloride with $1 \mu \mathrm{g} / \mathrm{kg}$ before anesthesia, while patients from group B were treated with the same dose of normal saline. In addition, there was an observation of the preventive effect of dexmedetomidine hydrochloride on postoperative neurological disorders in patients with liver cancer. The Medical Ethics Committee of the hospital had authorized this experiment. All patients had signed the informed consent forms and agreed to be included in this standard.

\subsection{Blind Restoration Algorithm of Ultrasound Image Based} on High-Order Spectral Variational Model. There were 2 parts in the medical ultrasound restoration method, mainly including the parameter estimation of the degraded medical ultrasound image point spread function (PSF) based on the high-order spectrum, and the estimation of applying the classic nondeconvolution blind restoration method to obtain the original image.

The PSF estimation algorithm for ultrasound images based on the high-order spectrum was to employ the feature that all high-order cumulants of the ultrasound envelope image signal were zero to eliminate the interference of Gaussian white noise and colored noise in non-Gaussian systems, thereby improving the accuracy of PSF estimation. For one-dimensional signals, the degradation model could be expressed in the following:

$$
g(n)=f(n) * h(n)+u(n) .
$$

In equation (1), $g(n)$ stands for an output signal. Assuming that the input signal $f(n)$ was stationary and nonGaussian, zero-mean, and same distributed, the system function $h(n)$ was invariant, and $u(n)$ was additive noise with zero mean. Therefore, $h(n)$ was estimated through the bispectrum transform, as shown in the following equation:

$$
h(n) \approx \operatorname{DET}^{-1}\{\exp (\operatorname{DET}(b(n)))\}
$$

The parameter identification method was selected for the estimation of medical ultrasound image PSF based on the highorder spectrum, and the autoregressive (AR), moving average 
(MA), and ARMA model of the time sequence were obtained according to the high-order statistics of the system output. The order of the ARMA model should be determined. Then, the high-order statistical information theory was applied to establish a system parameter identification method.

Based on the parameter estimation method of the ARMA model, the time original image was modeled as an AR process and the PSF model was changed into a moving average (MA) process, thereby representing the degraded image as a noisy observation of the AR moving average process. The ARMA model parameters were estimated to obtain the original clear image and the estimated value of PSF, which turned the blind image restoration into the determination of ARMA parameters.

2.3. Monitoring of Clinical Hemodynamic Indexes. The patients from the two groups received the routine hemodynamic monitoring before entering the operating room (T0), during surgery (T1), just after surgery (T2), and 1 hour after surgery (T3) in turn. The monitoring items included the average arterial blood pressure, heart rate, and BIS.

2.4. Detection of Immune and Liver Function Indexes. The detection of immune function indexes is as follows. Two venous blood samples (each with $2 \mathrm{~mL}$ ) were extracted from every patient before and after the surgery. A sample was placed in a blood collection tube with anticoagulant, and they should be mixed uniformly. The flow cytometry was employed to detect the $\mathrm{T}$ cell subsets, including $\mathrm{CD}^{+}, \mathrm{CD}^{+}$, and $\mathrm{CD} 8^{+}$. Besides, the ratio of $\mathrm{CD}^{+} / \mathrm{CD}^{+}$should be calculated. Moreover, the liver function indexes needed to be detected as follows. The other sample was placed in a vacuum blood collection tube and centrifuged, so as to take the upper serum. The enzyme-linked immunosorbent assay (ELISA) was adopted to determine the aspartate amino acid transferase (AST), alanine aminotransferase (ALT), and alkaline phosphate (ALP) in serums of all patients. All experimental operations were strictly in accordance with the kit instructions.

2.5. MoCA Score. The Montreal Cognitive Assessment (MoCA) Scale Chinese version was employed to examine the cognitive function of patients 24 hours after the surgery. The scale has a total of 30 scoring items, and each correct answer is counted as 1 point, and the total score is 30 points. If the research object has less than 12 years of education, 1 point should be added to the test score to correct for the research object's educational level deviation. If the score is greater than or equal to 26 points, the patient's cognitive function is considered normal. If the score is less than 26 points, it is considered that the patient has cognitive impairment. In order to ensure the accuracy of the test, at least two neurologists are required to measure and score the MoCA scale on all research objects during the test.

2.6. Monitoring of the Adverse Reaction Incidence. The patients from the two groups were investigated in terms of respiratory depression, nausea, and pain. What is more, the number of cases with adverse reactions was recorded after surgery, and the incidence of adverse reactions should also be calculated.

2.7. Statistical Analysis. All measurement data were represented as mean \pm standard deviation, and the data were statistically processed by SPSS 21.0 software package. Oneway analysis of variance (ANOVA) was adopted to analyze the comparison within groups, the comparison between groups was determined by $t$-test, and the comparison of count data was tested by the chi-square test. $P<0.05$ meant that there was a statistically substantial difference.

\section{Experimental Results}

3.1. Comparison on Abdominal Ultrasound Images of Liver Cancer Patients before and after Restoration Based on Ultrasound Image Restoration Algorithm. As shown in Figure 1, the images before and after restoration were compared, showing that the restored image was clearer than before. The experiment indicated that the method provided in this study could well preserve the edges of the image and effectively avoid the "blocking effect" in the smooth area of the image, thereby obtaining a higher-quality restored image, and the peak signal-to-noise ratio was improved.

3.2. Comparison on the Average Arterial Blood Pressure, Heart Rate, and Bispectral Index of Patients from the Two Groups at Different Time Points. Figure 2 reveals that the monitoring results of average arterial blood pressure in patients from group A during T1 and T3 were sharply lower than those of group B, and there was a statistically obvious difference $(P<0.05)$. Moreover, the perioperative average arterial blood pressure of patients from group A was all $60-65 \mathrm{mmHg}$, and the fluctuation range was small.

Figure 3 shows that the heart rates of patients in group $\mathrm{A}$ at T1, T2, and T3 were steeply lower than the rates of group $\mathrm{B}$, with a statistically great difference $(P<0.05)$. Besides, the recovery effect of the heart rate in patients from group A was better after surgery.

Figure 4 reveals that the BIS values of patients from group A reduced hugely during $\mathrm{T} 1$ in contrast to the values of group B $(P<0.05)$. However, the BIS of patients from group $B$ recovered better after the surgery.

3.3. Comparison on Immune and Liver Functions of Patients from the Two Groups before and after Surgery. Table 1 shows the test results of immune function in patients from group A and $\mathrm{B}$. The $\mathrm{CD}^{+}, \mathrm{CD}^{+}$, and $\mathrm{CD} 4^{+} / \mathrm{CD}^{+}$levels of patients from the two groups after surgery decreased sharply in contrast to those before surgery $(P<0.05)$. What is more, the levels of $\mathrm{CD}^{+}, \mathrm{CD}^{+}$, and $\mathrm{CD}^{+} / \mathrm{CD}^{+}$in patients from group A after surgery rose dramatically in contrast to those of group B $(P<0.05)$.

The test results of liver function are shown in Table 2. The AST, ALT, and ALP levels of patients from the two groups increased obviously after surgery on comparing with before 

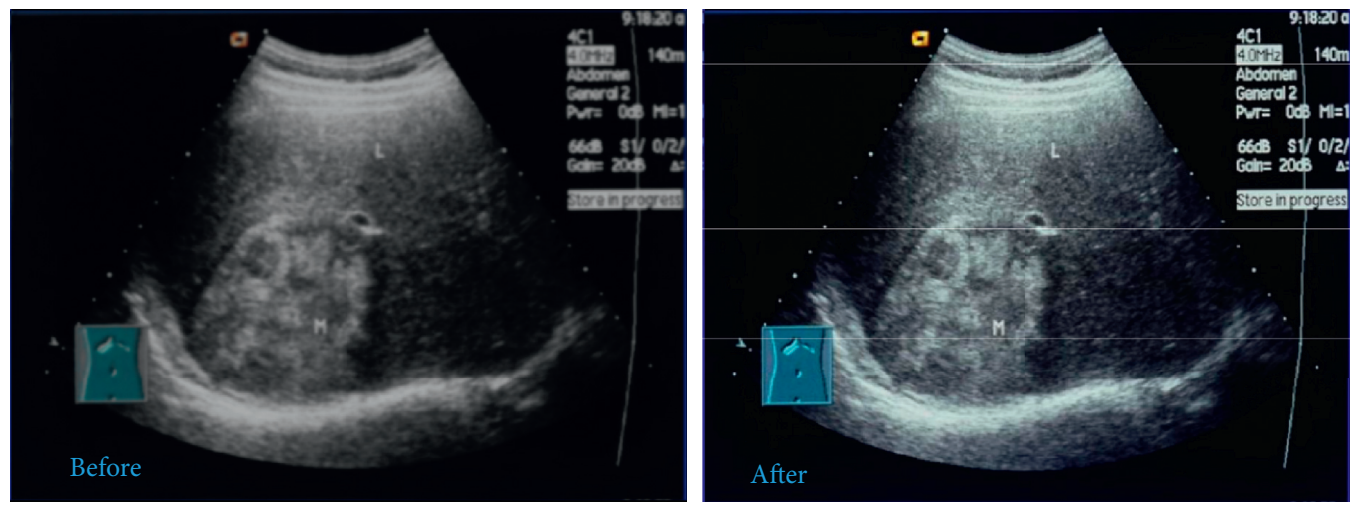

Figure 1: Comparison on the ultrasound images of the patient's abdomen before and after restoration.

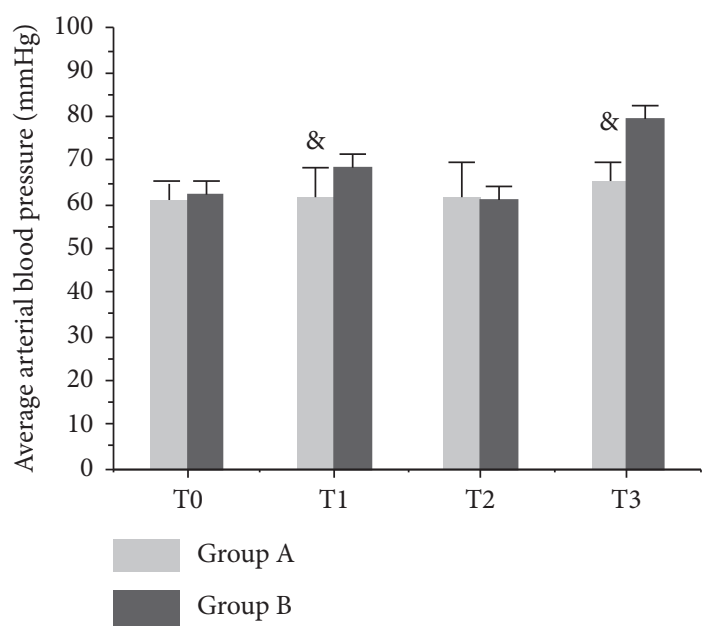

FIGURE 2: Comparison on the average arterial blood pressure in patients from both groups at different time points (note: $\&$ indicates that the difference was statistically marked in contrast to the control group $(P<0.05))$.

surgery $(P<0.05)$. Besides, the AST, ALT, and ALP levels of patients from group $B$ were markedly higher than the levels of group A $(P<0.05)$, and the difference was statistically remarkable.

\subsection{Comparison on MoCA Score and Cognition Dysfunction} Incidence of Patients from the Two Groups after Surgery. As shown in Table 3, the postoperative MoCA score of patients from group A was $27.51 \pm 2.02$ points, which was slightly lower than that before the surgery $(P>0.05)$. But the postoperative MoCA score of patients from group A was obviously less than the score of group $\mathrm{B}(P<0.05)$. There were 4 cases with cognition dysfunction from group $\mathrm{A}$, with an incidence of $12.91 \%$, while 10 cases suffered from cognition dysfunction from group B, and its incidence was $32.26 \%$. Thus, the incidence of patients from group A dropped extremely on comparing with group B $(P<0.05)$.

3.5. Comparison on the Adverse Reaction Incidence of Patients from Both Groups after Surgery. The number of cases with adverse reactions such as respiratory depression, nausea, vomiting, and pain from group A was lower than the number of group B (Table 4). The total incidence of adverse reactions in patients from group A was $6.45 \%$, which was hugely lower than the incidence of group B (22.58\%) $(P<0.05)$.

\section{Discussion}

Ultrasound imaging is an advanced medical diagnosis technology. It has good directionality, no invasion, no ionizing radiation, and strong real-time performance. Moreover, it has high sensitivity and resolution. Its diagnostic accuracy rate for small lesions is still high. Therefore, ultrasound imaging has been extensively applied in clinical diagnosis of liver cancer. What is more, an algorithm based on high-order spectral variational models was proposed in this study to degrade and restore ultrasound images because of the low resolution, so as to improve the image quality, which was consistent with the research results of Ong and Chandran [12]. However, the PSF parameters mentioned in this study were ideal, and the obtained parameters were employed to obtain the original clear image through the parameter estimation method of the ARNA model so that the blind image restoration was turned into the determination of the ARMA parameters. In actual ultrasound 


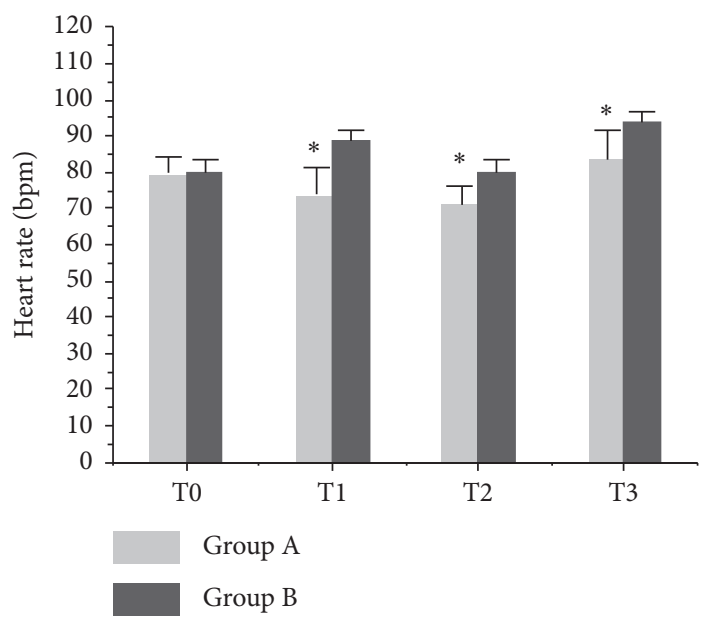

Figure 3: Comparison on the heart rates of patients from both groups at different time points (note: ${ }^{*}$ means that the difference was statistically substantial compared with the control group $(P<0.05))$.

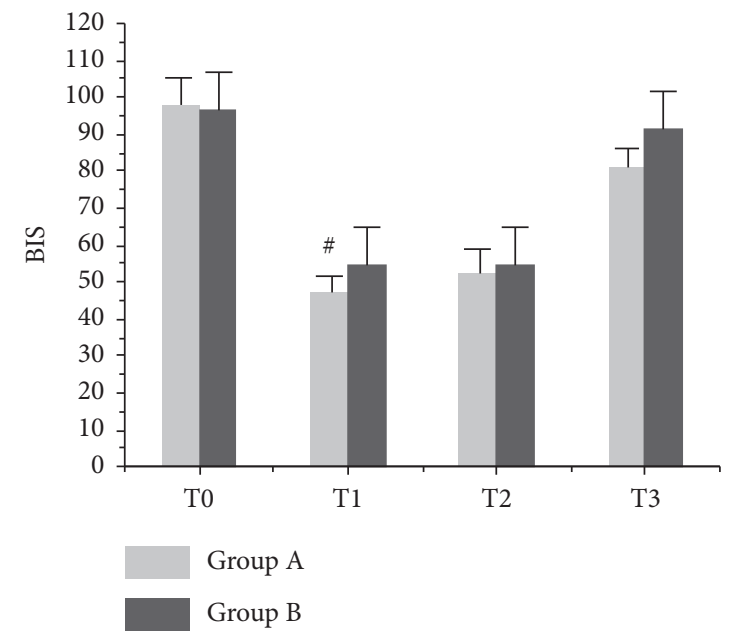

FIgURE 4: Comparison on BIS of patients from the two groups at different time points (note: \# means that the difference was statistically substantial in contrast to the control group $(P<0.05))$.

TABLE 1: Comparison on the immune function in patients from the two groups before and after surgery.

\begin{tabular}{lcccc}
\hline & Number of cases & $\mathrm{CD}^{+}(\%)$ & $\mathrm{CD}^{+}(\%)$ & $\mathrm{CD}^{+} / \mathrm{CD}^{+}$ \\
\hline Before surgery & - & $64.35 \pm 6.61$ & $42.39 \pm 5.27$ & $1.25 \pm 0.24$ \\
Group B after surgery & 31 & $46.78 \pm 7.14$ & $29.93 \pm 5.31$ & $1.04 \pm 0.21$ \\
Group A after surgery & 31 & $53.69 \pm 6.61^{*}$ & $33.83 \pm 6.58^{*}$ & $1.16 \pm 0.23^{*}$ \\
$t$ & - & - & - & $<$ \\
$P$ & - & $<0.05$ & $<0.05$ & $<0.05$ \\
\hline
\end{tabular}

Note: ${ }^{*}$ indicates that the difference was statistically obvious compared with group $\mathrm{B}(P<0.05)$.

imaging, PSF usually presents spatial variability due to various reasons such as focusing, diffraction, attenuation, and phase distortion. Tissue reflection signals, time gain amplifiers, and scattering space can all affect the recovery effect by influencing the signal-to-noise ratio. This was in accordance with the research results of Jan et al. [13]. There are few related studies at present, and further research and exploration are needed to improve the spatial coordinate transformation and restoration model.

Sevoflurane is a commonly used anesthetic in clinical surgery, but its side effects have strong respiratory depression [14]. Therefore, drug combination is often adopted to reduce the side effects of sevoflurane. Dexmedetomidine, as a sedative, has no inhibitory effect on breathing, which was 
TABle 2: Comparison on liver function of patients from the two groups before and after surgery (U/L).

\begin{tabular}{lcccc}
\hline & Number of cases & AST & ALT & ALP \\
\hline Before surgery & - & $18.75 \pm 6.15$ & $24.17 \pm 7.73$ & $60.15 \pm 18.14$ \\
Group B after surgery & 31 & $158.88 \pm 45.82$ & $189.78 \pm 57.59$ & $300.13 \pm 108.43$ \\
Group A after surgery & 31 & $98.76 \pm 32.86^{*}$ & $115.64 \pm 33.95^{*}$ & $211.25 \pm 77.79^{*}$ \\
$t$ & - & - & - & - \\
$P$ & - & $<0.05$ & $<0.05$ & $<0.05$ \\
\hline
\end{tabular}

Note: ${ }^{*}$ means that there was a statistically great difference in contrast to group $\mathrm{B}(P<0.05)$.

Table 3: Comparison on MoCA score and cognition dysfunction incidence of patients in the two groups.

\begin{tabular}{lcc}
\hline & MoCA & POCD \\
\hline Before surgery & $28.12 \pm 1.45$ & - \\
Group B after surgery & $25.79 \pm 2.35$ & $32.26 \%$ \\
Group A after surgery & $27.51 \pm 2.02^{*}$ & $12.91 \% *$ \\
$t$ & - & - \\
$P$ & $<0.05$ & $<0.05$ \\
\hline
\end{tabular}

Note: ${ }^{*}$ shows that there was a statistically obvious difference in contrast to group $\mathrm{B}(P<0.05)$.

TABLE 4: Statistics of adverse reactions in patients from the two groups.

\begin{tabular}{lccccc}
\hline Group & Number of cases & Respiratory depression & Nausea and vomiting & Pain & Total incidence \\
\hline Group A & 31 & 1 & 1 & 0 & $2(6.45)^{*}$ \\
Group B & 31 & 3 & 2 & 2 & $7(22.58)$ \\
& - & - & - & - & 4.71 \\
$P$ & - & - & - & - & 0.032 \\
\hline
\end{tabular}

Note: ${ }^{*}$ shows that the difference was statistically considerable in contrast to group B $(P<0.05)$.

in line with the research findings of Zhang et al. [15]. The anesthesia recovery period is one of the key aspects for treatment during anesthesia of patients, and it is also the more dangerous stage of the patient's perioperative period. Thus, the clinical indexes of the patient after surgery should be closely monitored to ensure that the patient passes the dangerous period. In this study, the high-order spectral variation model was constructed to blindly restore the ultrasound image. Then, there was a comparison on the ultrasound images of patients from group A (with the treatment of dexmedetomidine combined with sevoflurane) and group B (with the treatment of sevoflurane). The results showed that the UIR based on high-order spectral variational models could promote the image quality. There were no much differences in the perioperative changes of average arterial blood pressure and heart rate in patients from group A before and after surgery, while the changes of patients from group B were greatly different from those before surgery, indicating that the difference was statistically marked $(P<0.05)$. The combined application of dexmedetomidine, sevoflurane, and intraoperative anesthesia could reduce the amount of sevoflurane, thereby reducing the respiratory inhibition of patients, which was consistent with the findings of Goyagi [16]. The postoperative immune function indexes $\left(\mathrm{CD}^{+}, \mathrm{CD}^{+}\right.$, and $\left.\mathrm{CD} 4^{+} / \mathrm{CD}^{+}\right)$of patients from group A were lower than those before the surgery, but the reduction of those indexes in patients from group $A$ was smaller than the reduction of group $\mathrm{B}(P>0.05)$. Besides, the liver function indexes (AST, ALT, and ALP) of patients from group A elevated markedly in contrast to those before the surgery $(P<0.05)$. Nervous disorders in the postoperative recovery period are mainly due to the action of general anesthetics on the nerve center, but the inhibitory effect on the center is different. In most cases, the function of the higher brain center is not fully recovered after the patient's consciousness is restored, which affects the patient's sensory response and processing $[17,18]$. The results of the study found that BIS increased by a wider margin in the single sevoflurane group versus the dexmedetomidine hydrochloride combined with sevoflurane group $(P<0.05)$. The postoperative MoCA score of patients from group A increased obviously in contrast to the score of group B $(P<0.05)$, and the incidences of cognition dysfunction and adverse reactions in group A reduced hugely compared with group $\mathrm{B}(P<0.05)$. It suggested that the anesthesia treatment of dexmedetomidine hydrochloride combined with sevoflurane had better anesthesia effect and less impact on patients.

\section{Conclusion}

It was found that UIR based on high-order spectral variational models could promote the quality of ultrasound images and enhance resolution. The combination of sedative dexmedetomidine and sevoflurane was applied in the surgery, which could effectively maintain the patient's perioperative hemodynamic parameters and reduce the gap between the patient's intraoperative and postoperative 
hemodynamic parameters and preoperative parameters. In addition, the combined medication elevated the growth of immune and liver function indexes, so as to effectively inhibit the incidence of postoperative cognitive dysfunction and adverse reactions in patients. The results of this study provide a reference value for the theory that the combined use of UIR, sedatives, and anesthetics can prevent patients from neurological disorders during the recovery period, so as to accelerate the recovery process of patients after surgery. However, the perioperative liver cancer patients are only selected as the research objects in this study, and the sample size is small, which has certain limitations. In the future research, the research objects should be expanded to surgery for other diseases, and the number of samples should be increased in order to strengthen the persuasiveness of the data. Thus, it can better explain the preventive effects of the combined application of dexmedetomidine and sevoflurane on neurological disorders during the recovery period of anesthetized patients, so as to better exert its clinical application value.

\section{Data Availability}

No data were used to support this study.

\section{Conflicts of Interest}

The authors declare that they have no conflicts of interest.

\section{References}

[1] D. Maucort-Boulch, C. de Martel, S. Franceschi, and M. Plummer, "Fraction and incidence of liver cancer attributable to hepatitis B and C viruses worldwide," International Journal of Cancer, vol. 142, no. 12, pp. 2471-2477, 2018.

[2] S. T. Orcutt and D. A. Anaya, "Liver resection and surgical strategies for management of primary liver cancer," Journal of the Moffitt Cancer Center, vol. 25, no. 1, 2018.

[3] B. J. A. Palanca, M. S. Avidan, and G. A. Mashour, "Human neural correlates of sevoflurane-induced unconsciousness," British Journal of Anaesthesia, vol. 119, no. 4, pp. 573-582, 2017.

[4] K. G. M. Dexmedetomidine, "A review of its use for sedation in the intensive care setting," Drugs, vol. 75 , no. 10, pp. 1119-1130, 2015.

[5] C. R. M. Barends, A. Absalom, B. van Minnen, A. Vissink, and A. Visser, "Dexmedetomidine versus Midazolam in procedural sedation. A systematic review of efficacy and safety," PloS One, vol. 12, no. 1, Article ID e0169525, 2017.

[6] J. Afonso and F. Reis, "Dexmedetomidine: current role in anesthesia and intensive care," Brazilian Journal of Anesthesiology, vol. 62, no. 1, pp. 118-133, 2012.

[7] Y. Punjasawadwong, A. Phongchiewboon, and $\mathrm{N}$. Bunchungmongkol, "Bispectral index for improving anaesthetic delivery and postoperative recovery," The Cochrane Database of Systematic Reviews, vol. 2014, no. 6, Article ID CD003843, 2014.

[8] S. G. Memtsoudis, C. Cozowicz, M. Nagappa et al., "Society of anesthesia and sleep medicine guideline on intraoperative management of adult patients with obstructive sleep apnea," Anesthesia \& Analgesia, vol. 127, no. 4, pp. 967-987, 2018.
[9] A. Lehn, J. Gelauff, I. Hoeritzauer et al., "Functional neurological disorders: mechanisms and treatment," Journal of Neurology, vol. 263, no. 3, pp. 611-620, 2016.

[10] P. N. Wells, H. D. Liang, and T. P. Young, "Ultrasonic imaging technologies in perspective," Journal of Medical Engineering \& Technology, vol. 35, no. 6-7, pp. 289-299, 2011.

[11] M. Cüneyitoğlu Özkul, Ü. E. Mumcuoğlu, and İ. T. Sancak, "Single-image bayesian restoration and multi-image superresolution restoration for b-mode ultrasound using an accurate system model involving correlated nature of the speckle noise," Ultrasonic Imaging, vol. 41, no. 6, pp. 368-386, 2019.

[12] H. Ong and V. Chandran, "Identification of gastroenteric viruses by electron microscopy using higher order spectral features," Journal of Clinical Virology, vol. 34, no. 3, pp. 195-206, 2005.

[13] M.-L. Jan, M.-W. Lee, and H.-M. Huang, "PSF reconstruction for Compton-based prompt gamma imaging," Physics in Medicine \& Biology, vol. 63, no. 3, Article ID 035015, 2018.

[14] J.-M. Constantin, A. Momon, J. Mantz et al., "Efficacy and safety of sedation with dexmedetomidine in critical care patients: a meta-analysis of randomized controlled trials," Anaesthesia Critical Care \& Pain Medicine, vol. 35, no. 1, pp. 7-15, 2016.

[15] X. Zhang, D. Wang, M. Shi, and Y. Luo, "Efficacy and safety of dexmedetomidine as an adjuvant in epidural analgesia and anesthesia: a systematic review and meta-analysis of randomized controlled trials," Clinical Drug Investigation, vol. 37, no. 4, pp. 343-354, 2017.

[16] T. Goyagi, "Dexmedetomidine reduced sevoflurane-induced neurodegeneration and long-term memory deficits in neonatal rats," International Journal of Developmental Neuroscience, vol. 75, no. 1, pp. 19-26, 2019.

[17] F. Chung, S. G. Memtsoudis, S. K. Ramachandran et al., "Society of anesthesia and sleep medicine guidelines on preoperative screening and assessment of adult patients with obstructive sleep apnea," Anesthesia and Analgesia, vol. 123, no. 2, p. 1, 2016.

[18] M. Mulder and R. G. Geocadin, "Neurology of cardiopulmonary resuscitation," Handbook of Clinical Neurology, vol. 141, pp. 593-617, 2017. 